\title{
The sound of silence: global health challenges from the front
}

Winner

(Medical student category)
T

here are no sirens. Nor is the silence disrupted by the rush of feet or frantic blue lights.

Instead, our enduring patients arrive at the hospital gates on foot, most having travelled for many hours, if not days, through the unrelenting $40^{\circ} \mathrm{C}$ heat; the privileged cling to motorcycles already laden with firewood and kitchen utensils in anticipation of a prolonged admission.

Through these same gates arrived Abakar (name changed to maintain confidentiality), so weakened by the ruthless combination of tuberculosis, suspected HIV, diarrhoea and malnutrition that he was unable to hold his fragile frame from the ground. At two-and-a-half years, his weight was that of a 4-month-old baby.

He passed away early this morning.

I have just heard the news and am overwhelmed.

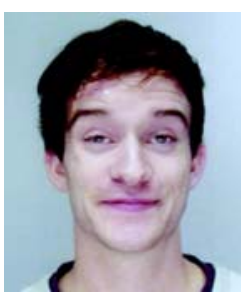

James D Smith Fourth Year Medical

Student

Newcastle University, Newcastle upon Tyne, UK. J.D.SMITH3@NCL.AC.UK doi: 10.5694/mjal2.10783
Yet the ward round must continue. There is little time to reflect. Not now at least. Eighty patients await their daily review, while the queue requesting consultation with the only doctor for many miles now curves away from her office.

A young man whose limbs are mottled with Kaposi's sarcoma requires urgent antiretroviral treatment. On the opposite bed sits an elderly man whose neglected diabetic ulcer has progressed to advanced necrosis. He has just refused the amputation that might save his life. By the doorway, a man unwraps his bandaged hand to reveal a seeping nodular mass. A chronic wound left for 18 months has developed into an extensive squamous cell carcinoma.

In the Salle d'Isolement, three patients, including a 9-month-old infant, receive treatment for drug-resistant tuberculosis. The protocol lasts 8 long months and requires strict compliance and regular review. Should the treatment 


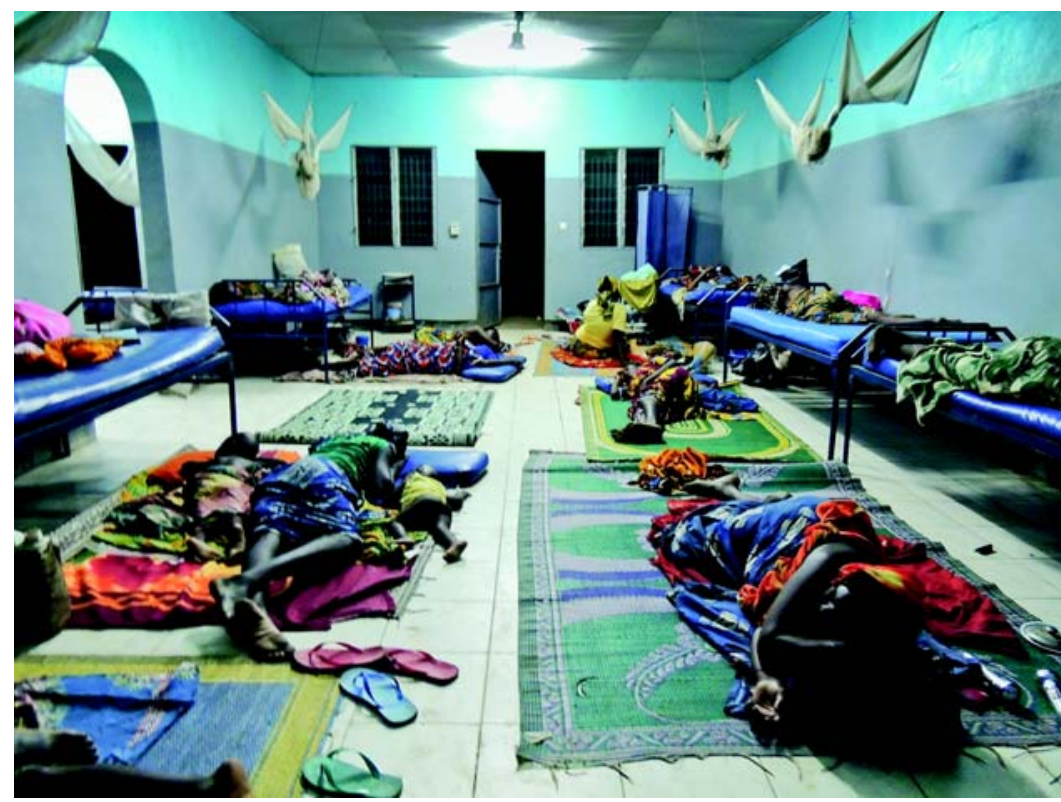

In the crowded Département de Pédiatrie, mothers and their children relocate indoors with the start of the seasonal rains

fail, there is no second-line intervention. Along the opposite terrace, it is possible to smell the latest outbreak of measles before you see the long line of lethargic children: a local remedy consists of smearing the skin of those infected with peeled onions. The disease has been endemic here for generations and, while the vaccination coverage in this district is relatively high, dangerously low rates across the permeable border with Nigeria continually risk igniting an epidemic.

Under the sparse shade offered by the neem trees, this same corner of the compound is intermittently transformed into a makeshift cholera camp. Two years ago, a catastrophic epidemic of the disease spiked with 1600 identified cases. We do not expect the outbreak to entice the humanitarian organisations again this year, but further cases are inevitable.

The Département de Pédiatrie has reached capacity. A young girl recovers from an Echis snake bite; the parallel incisions at the site are a sign that she was seen by the local healer first. A vial of antivenom makes up for lost time, and reverses the widespread anticoagulative effect of the otherwise lethal venom. The heat has caused a threefold increase in the incidence of snakebites, and we will exhaust our stock of vials next week. The pharmaceutical company has temporarily discontinued production, and we are unable to find an alternative supplier.

In the adjoining room, an infant is introduced with a single word: "kwashiorkor". Thankfully, she is recovering well and has lost the deceptive fullness of oedema. On the adjacent bed is propped a child whose marasmic features disguise her young age. She mutely opens and closes her mouth, too weak to produce a sound. Her bright wax-print blanket is incongruous as the backdrop to the frightening state of her entirely preventable condition.

Abakar's bed lies empty.

In the overflow ward, a mother and child have been admitted together. The mother's untreated epilepsy precipitated a seizure over the cooking fire. Her son, strapped to her back, suffered the same third-degree burns that she did. The daily debridement and application of honey dressings are agonising, but we lack the appropriate anaesthesia to numb the pain.
A young woman arrives at the hospital in labour. For a dangerous 3 days she was kept at home, becoming progressively weaker. As she is carried to the table, the baby's arm can be seen protruding from between her legs. There is no doubt that she needs an immediate caesarean section, yet the family refuse on her behalf and insist on leaving the hospital.

Relying solely on clinical judgement and the reassuring amplification of the Pinard stethoscope, three women have just delivered healthy babies. A shortage of examination gloves means we now recycle each and every pair. When they are worn through, we save the rubber bands at the wrist and use them to tie off the umbilical cord. The daily resourcefulness of my colleagues is inspiring, but further underscores a chronic insufficiency of the most essential resources.

In the Département de Chirurgie a young man is treated for a gunshot injury that shattered his right knee. The lights flicker before the invaluable generator again illuminates his open wound. A recent outbreak of violence across the border has strained the team and frozen our transport of biopsy specimens to the Nigerian pathologist. The man's operation is overseen, not by an extended team of anaesthetists and orthopaedic surgeons, but by a contingent of the countless lizards with whom we share the hospital compound. In this heat, even our coldblooded companions search for the relative cool of the operating room.

With the operation complete, the patients continue to arrive. Only as the sun begins to set over the sparse plains and compact mud-and-thatch villages of this corner of the Sahel, will the number of new arrivals begin to subside.

As I write, the four muted grey walls of our desert health outpost are slowing turning a dusty amber, and the hospital has lulled into a deceptively tranquil state. Women and children collect water from the central well, while the smell of boiling bouillie counters the acrid smoke that rises from the deep waste disposal pit.

It is here, at the grassroots level, working with front-line health services in the most resource-deprived region of a resource-deprived country, that our patients collectively personify a multitude of the most urgent global health challenges. There is no isolated textbook solution. Instead, we attempt to understand these challenges in the complex context in which they arise: the chronic insufficiency of adequately trained staff; the widely held belief that breastfeeding has caused an infant's febrile state, subsequently triggering a decline into malnutrition and further ill health; the extortionate rise in the price of life-saving oral rehydration sachets imposed by the Ministry of Health; international agencies seemingly more concerned with quick results than investment in sustainable health system development; generations of poverty and food insecurity in our region; and a government with one of the world's highest and most infamous corruption ratings. The daily triage and prioritisation of such issues is all that keeps the hospital from sinking into the sand.

I look now at Abakar's tattered and muddy carnet de santé, and his remaining three sealed syringes, and cannot hold back the sadness and frustration. In the absence of a united and determined outcry, what chance did he have?

This silence must be broken. 